\title{
Contextual effects on metaphor comprehension in reading
}

\author{
ALBRECHT WERNER INHOFF, SUSAN D. LIMA, and PATRICK J. CARROLL \\ University of Massachusetts, Amherst, Massachusetts
}

\begin{abstract}
Subjects read target sentences preceded by either short or long context that induced either a metaphoric or a literal target reading. As had been found by Ortony, Schallert, Reynolds, and Antos (1978), metaphoric targets were comprehended about as quickly as literals when context was long, but more slowly than literals when context was short. The latter result may have been due to the failure of computing a conceptual relationship between short context and metaphoric target; targets unrelated to prior context took as long to comprehend as metaphoric targets. Another experiment showed that metaphorically expressed targets were read more quickly when they followed metaphorically expressed context than when they followed literal context, but literal targets were read quickest when they followed literal context. These results are discussed within a schema framework and within a "process priming" hypothesis.
\end{abstract}

Metaphors are hypothesized to have arisen at the very beginning of the conceptual evolution of language (Cassirer, 1923). Despite the importance of metaphors in language comprehension, relatively few theoretical and empirical enterprises have set out to explore figurative language. Early psycholinguistic theories explained metaphor comprehension within the associationist view. For example, Koen (1965) assumed that the literal and metaphoric interpretations of a word are established through linking verbal associations. In particular, Koen hypothesized that a word's metaphoric reading is derived from its literal reading through a search for common associations. Verbrugge and McCarrell (1977) rejected this associationist claim. They hypothesized that people infer a metaphoric meaning when it becomes apparent that a literal interpretation is unsatisfactory. This hypothesis was tested in a series of elegant studies in which subjects heard only one of a pair of metaphoric sentences about the same topic:

(1) Billboards are warts on the landscape. (are ugly protrusions on a surface)

(2) Billboards are the yellow pages of a highway. (tell you where to find business in the area)

As in Richards (1936), billboards is referred to as "topic," and warts and yellow pages are "vehicles." The paraphrases in parentheses, called the "grounds," were

This research was supported by National Science Foundation Grant BNS 79-17600 and Grant HD17246 from the National Institute of Health (principal investigator, Keith Rayner). Susan Lima is now at the Center for Cognitive Science, M.I.T. Patrick Carroll is now at the Center for Cognitive Science, University of Texas at Austin. We thank Steven Keele, Janet Randall, Maria Slowiaczek, Keith Rayner, and two anonymous reviewers for their valuable comments. Requests for reprints should be sent to Albrecht Werner Inhoff, Neuropsychology Laboratory, Department of Neurology, Good Samaritan Hospital, 1015 NW 22 Avenue, Portland, OR 97210.

-Steven W. Keele served as Action Editor on this manuscript. not presented to subjects. In a recall task, it was found that relevant grounds (those that paraphrased the presented metaphoric sentence) were better recall cues than nonrelevant grounds (those that paraphrased the unpresented member of the metaphoric sentence pair). It was concluded that subjects must have inferred the relevant ground during the comprehension of the presented metaphoric sentence.

Although recall tasks assess the product of comprehension, these tasks do not necessarily reflect computational operations as they occur during reading. To gain a more immediate look at the encoding of metaphoric and literal sentences, Ortony, Schallert, Reynolds, and Antos (1978) recorded sentence reading times. They hypothesized that how a metaphoric target sentence is processed depends on the sufficiency of the conceptual framework established by prior context. If prior context fails to make apparent the metaphoric interpretation of a target sentence, as is often the case with rather short context, then readers will tend to initially obtain the literal interpretation of the metaphoric target sentence. According to the stage model of Searle $(1969,1979)$, this interpretation is checked against prior context. If the literal interpretation conflicts with prior context, then additional inference strategies yield a metaphoric reinterpretation of the target sentence. On the other hand, if prior context establishes a conceptual framework that strongly suggests the metaphoric interpretation of a metaphoric target sentence, as is often the case with rather long context, then the metaphoric interpretation becomes automatically available without need for a reinterpretation stage. For example, consider the target sentence.

(3) The hens clucked noisily,

in which hens can be literally interpreted as farm animals or metaphorically interpreted as chatting women. Both the 
literal and metaphoric interpretations are apparent when the target sentence is read with long prior context:

(4) In the back of the barn, the farmer's youngest child gathered pebbles and skipped them deftly across a puddle by the chicken coop. He knew that he was supposed to be feeding the animals but he kept on flicking at the birds. The hens clucked noisily.

(5) At a meeting of the women's club the youngest member requested the floor and brought up the issue of supporting the equal rights amendment. The importance of the issue outweighed her discomfort in speaking before the group. They reacted as she expected. The hens clucked noisily.

Literal interpretation remains fairly unimpaired when contextual support is decreased [see (6)]; in contrast, metaphoric interpretation becomes difficult when only short prior context is provided [see (7)].

(6) In the back of the barn, the hens clucked noisily.

(7) At a meeting of the women's club, the hens clucked noisily.

On the basis of their view of metaphor comprehension, Ortony et al. (1978) predicted that when short prior context is provided, interpreting a target sentence metaphorically is more time consuming than interpreting it literally. However, when long context is provided, no reinterpretation stage is needed, and therefore metaphoric interpretation should not be more time consuming than literal interpretation. Consistent with these predictions, Ortony et al. observed longer target sentence reading times when a metaphoric interpretation was required than when a literal interpretation was required only in the short-context condition. The long-context condition yielded comparable reading times for literal and metaphoric targets.

However, the empirical support for the view of Ortony et al. (1978) remains inconclusive. Although their results do suggest that ample contextual support obviates the need for multiple stages in metaphor comprehension, they leave unanswered the question of whether or not the stage model applies even when context is scanty. Sentence reading time in the metaphor condition may have been longer than in the literal condition under short context, not because of a successful metaphoric reinterpretation stage, but because subjects failed to generate any satisfactory target interpretation. After repeated unsuccessful attempts to integrate the literal meaning of the target with prior context, subjects might eventually conclude that the two statements must simply be unrelated. Short prior context may often be insufficient to generate any plausible metaphoric interpretation of the target sentence. For example, readers may never interpret hens as women in the target expression in context (7). A lack of apparent relationship between literal context and metaphoric target is even more evident in (8) and (9), where (9) is the metaphoric target:

(8) The children continued to annoy their babysitter.

(9) Regardless of the danger, the troops marched on.

In addition, the claim that metaphoric and literal targets with long context did not differ in reading time may not be justified. Under long prior context, literal sentences were read approximately $100 \mathrm{msec}$ faster than metaphoric sentences. This difference nearly reached significance $[F(1,60)=2.73, p<.15]$, and it is large enough to question acceptance of the null hypothesis. Moreover, the relatively fast reading time in the long-context condition may have exerted a floor effect, obscuring an actual difference in processing time.

The first two experiments reported here addressed these two issues. We used the passages used by Ortony et al. (1978), which consisted of a target sentence preceded by long or short literal-inducing or metaphor-inducing context. In addition, we included an "unrelated" condition, in which contexts and target sentences were paired randomly. Based on Ortony et al., we predicted that in the short-context experiment (Experiment 1), metaphoric sentences would take longer to read than literal sentences. Similarly, unrelated target sentences should take longer to read than literal sentences. In both instances, subjects are expected to reject an initial literal interpretation of the target sentence and to attempt a (metaphoric) reinterpretation. When such a reinterpretation remains inadequate, as it would in the unrelated condition, subjects may either attempt additional reinterpretations of the target or conclude that context and target are unrelated. If repeated reinterpretations do occur in the unrelated condition, then longer reading times should be observed for the unrelated than for the metaphoric target sentences. If, on the other hand, subjects conclude that the sentences are unrelated, then no reinterpretations of the unrelated target occur, and no difference in the reading times of unrelated and metaphoric sentences should be observed.

The introduction of the unrelated controls also permits us to assess effects in the long-context experiment (Experiment 2). If high contextual constraint results in automatic metaphoric interpretation of metaphoric sentences, then we should replicate the finding that metaphoric sentences do not require longer reading times than literal sentences. Unrelated sentences should take longer to read than literal sentences and metaphoric sentences. However, if long prior context drastically decreases reading time, thus reducing the difference between literal and metaphoric sentence reading times, then literal and metaphoric sentence reading times may not differ reliably from sentence reading time in the unrelated condition. In addition, to gain insight into the on-line processing of metaphors, we measured readers' eye movements, allowing us to determine the time spent reading the target word itself [e.g., hens in (3) or troops in (9)].

\section{EXPERIMENTS 1 AND 2}

\section{Method}

\section{Subjects}

Twenty-four students at the University of Massachusetts were paid to participate, 12 in Experiment 1 and 12 in Experiment 2. All subjects had normal vision. 


\section{Design}

Similarly to Ortony et al. (1978), we measured the time it took to read a target sentence under different conditions of target interpretation (literal, metaphoric, and unrelated). Three lists were constructed, each list containing the same targets in the same serial order. The three lists differed, however, with respect to the prior context. Context consistent with literal target interpretation in one list was replaced with context consistent with metaphoric interpretation in the second list, and with unrelated context in the third list. Each list contained five passages in each of the three conditions (literal, metaphoric, and unrelated), and the order of conditions was counterbalanced across lists. Each subject was randomly assigned to one list. In the statistical analyses, list was treated as a between-subjects factor; target interpretation was considered a withinsubject factor.

\begin{abstract}
Materials
We used the stimulus materials of Ortony et al. (1978) under shortcontext (Experiment 1) and long-context (Experiment 2) conditions. It was necessary to very slightly modify some of the passages in order to fit the demands of the display apparatus, which resulted in slightly shorter contexts than those that Ortony et al. (1978) employed. In the present experiments, short contexts ranged from 4 to 10 words in length (mean $=6.5$ ), whereas long contexts ranged from 31 to 57 words (mean $=43.9$ ). An experimental item consisted of a target sentence that was to be interpreted either literally or metaphorically, contingent on the preceding context [see examples (4), (5), (6), and (7)], or which was unrelated to the preceding context. Context and target sentences were presented successively in separate displays of text. The effectiveness of prior context in inducing a literal or metaphoric target interpretation was assessed by Ortony et al. in a separate normative study (see Ortony et al. for details). In the present experiments, unrelated context-target passages were created by randomly pairing contexts and targets that were not paired in the literal or metaphoric conditions. In instances in which random combinations happened to yield a conceptual relationship between context and target, an arbitrary regrouping ensured that context and target remained unrelated. Fifteen of the 16 items used by Ortony et al. were used in the present experiments, so that in a list, five sentences appeared in each of the three interpretation conditions. (Ortony et al. presented eight target sentences in each of their two interpretation conditions.)
\end{abstract}

\section{Apparatus}

The subject's eyes were $46 \mathrm{~cm}$ from a Hewlett-Packard 1300 A cathode ray tube (CRT) that was used to present the text. Three character spaces of text equaled $1^{\circ}$ of visual angle, and the stimuli were presented in lowercase. A black theater gel covered the screen so that the stimuli appeared clear and sharp. The CRT was adjusted to a comfortable brightness level for each subject. The luminance was occasionally reduced during the experiment because of pupillary constrictions that led to track losses during the eye movement recording.

Eye movement recording was accomplished by using a Stanford Research Institute Dual Purkinje Eyetracker. The eye tracker has a resolution of $10^{\prime}$ of arc and the output is linear over the visual angle that was occupied by each sentence. The eye tracker and the CRT were interfaced with a Hewlett-Packard $2100 \mathrm{~A}$ computer that controlled the experiment. The signal from the eye tracker was sampled every millisecond by the computer. Each $4 \mathrm{msec}$ of eye tracker output was compared to the output of the prior $4 \mathrm{msec}$ to determine whether the eye was fixated or in motion. The computer kept a complete record of the duration, sequence, and location of each fixation.

\section{Procedure}

The subjects were tested individually. When the subject arrived, a bite bar, which served to reduce head movements during the experiment, was prepared. Each subject received instructions about the procedure and was familiarized with the equipment. No information about the exact nature of the relationship between context and target was provided. A calibration of the eye tracking system began each session. (For details, see Rayner, Inhoff, Morrison, Slowiaczek, \& Bertera, 1981.)
Experiment 1. In Experiment 1, a one-dimensional calibration (left to right) was performed. After the calibration, three crosses were displayed, at the right, center, and left of the screen. The subject's fixation point was marked by a fourth cross that moved in synchrony with his or her eyes. Text was read in the following manner: A cross appeared at the left side of the CRT. This position coincided with the first letter of a line of text. Text was displayed by the experimenter as soon as the reader's fixation marker was superimposed on the left-hand cross. The first line of text remained on the CRT until the subject pushed a button that replaced the line of text with the cross at the left side of the screen. Upon fixation of the cross, the experimenter displayed a second line of text. In each instance, context and target sentences appeared on separate lines of text. The cycle continued until the entire passage had been read.

Experiment 2. Experiment 2 was initiated with a two-dimensional calibration (horizontal and vertical) that yielded accurate eye tracking in both the vertical and horizontal dimensions. Two fixation crosses were presented, at the left- and right-hand top of the CRT. After the subject's fixation position was placed on the left-hand cross, a passage of text, ranging from 3 to 10 lines, was presented. The subject read the passage and then pressed a button. This buttonpress replaced the passage with a single line of text that contained the target. The subject pressed the button again after the target sentence had been read, which replaced the target with the fixation markers.

\section{Data Analysis}

Two different dependent measures were used: sentence reading time and total viewing time of critical words. Sentence reading time was calculated by adding the durations of all fixations on a sentence. Total viewing time was computed for critical words to explore the processing of those words assumed to be the focus of metaphoric reinterpretation. Total viewing time consists of the entire amount of fixation time spent on a particular word, including its initial reading and any rereading time resulting from regressive eye movements. This measure was used by Inhoff (1983) and was found to be particularly sensitive to readers' contextdependent word interpretation.

\section{Results and Discussion}

\section{Experiment 1: Short Prior Context}

The results of Experiment 1 revealed that literal sentences required an average reading time of $1,872 \mathrm{msec}$, whereas sentences in the metaphoric and unrelated conditions required 2,039 and $2,015 \mathrm{msec}$, respectively $[F(2,18)=5.25, p<.025]$. Newman-Keuls tests indicated that sentences were read significantly faster when they were to be interpreted literally than when they were to be interpreted metaphorically $(\mathrm{q}=4.25, \mathrm{p}<.05)$ or when they were unrelated to the prior context $(q=3.64$, $\mathrm{p}<.05$ ). The difference in sentence reading time in the metaphoric and unrelated conditions was not reliable. Total viewing time data followed a similar pattern: There were shorter total viewing times on critical words in the literal $(333 \mathrm{msec})$ than in the metaphoric $(414 \mathrm{msec})$ or unrelated $(419 \mathrm{msec})$ conditions. However, this effect failed to reach significance $[F(2,18)=1.51, p<.20]$.

This pattern of results replicates the Ortony et al. (1978) findings in showing that readers spent more time reading a target sentence in the metaphoric than in the literal condition if only short prior context was provided. Yet, the present results leave unanswered the question of whether short context actually induced a metaphoric reinterpretation of the target sentence. Since unrelated target sentences 
did not require longer reading times than metaphoric target sentences, it seems plausible that both types of targets were internally represented as unrelated sentences. However, the present results could be seen as consistent with the Ortony et al. conclusion if it is assumed that only one metaphoric reinterpretation is attempted, which is either successful (so that the target is interpreted metaphorically) or unsuccessful (so that the target is perceived as unrelated and no further reinterpretations are invoked). The assumption of one attempted reinterpretation could lead to equivalent reading times for metaphoric sentences and unrelated sentences.

\section{Experiment 2: Long Prior Context}

Unlike the results in the short-context experiment, literal and metaphoric sentences under long context required similar amounts of reading time $(2,127$ and $1,985 \mathrm{msec}$, respectively), both of which were considerably shorter than the sentence reading time in the unrelated condition $(2,611 \mathrm{msec})$. Newman-Keuls tests indicated no reliable difference between the sentence reading times in the literal and metaphoric conditions $(q=1.78, p>.05)$, both of which were reliably shorter than the sentence reading time in the unrelated condition $(q=6.08, p<.01 ; q=7.87$, $p<.01)$. A similar pattern was evident in the total viewing time of critical words. Literal and metaphoric critical words received an average total viewing time of 443 and $457 \mathrm{msec}$, respectively, which were somewhat shorter than the total viewing times of critical words in the unrelated condition $(492 \mathrm{msec})$. However, as in the analysis of total viewing time in the short-context experiment, the differences in total viewing time were not statistically reliable $[\mathrm{F}(2,18)=1.70, \mathrm{p}<.20]$.

We have thus successfully replicated the Ortony et al. (1978) finding of no reliable difference between literal and metaphor sentence reading times when long prior context was provided. In addition, the present results suggest that the lack of a significant difference was not simply due to a floor effect, caused by relatively fast reading of the target under long context, because the unrelated targets took significantly more time to read than either the metaphoric or literal targets. With sufficient contextual support, metaphors are comprehended as efficiently as literals.

\section{EXPERIMENT 3}

The results of Experiments 1 and 2 showed that a readily apparent conceptual relationship between target and context was crucial to successful metaphoric processing. When long context supported the metaphoric sense of a target sentence, metaphoric interpretation proceeded as swiftly as did literal interpretation of the same sentence when its literal sense was supported by prior context. On the other hand, when context was short and the conceptual relationship between context and target was not so readily apparent, readers may have failed to metaphorically interpret target sentences within the frame of prior context.

An issue that remains unanswered by the results of Experiment 1 and the results of Ortony et al. (1978) is whether or not length of context, per se, is critical to metaphor interpretation. It may be that short context can support metaphoric interpretation of a sentence, even if the short context used in Experiment 1 did not induce successful metaphoric target interpretation. An issue that remains unanswered in Experiment 2 is whether metaphoric and literal processes are structurally equivalent. Even though the finding that metaphor processing occurred in about the same amount of time as literal processing (Experiment 2) suggests that metaphor processing is essentially similar to literal processing, it may instead be the case that metaphors invoke a comprehension process different from that invoked by literals, but both the metaphor comprehension process and the literal comprehension process require about the same amount of time for completion. Furthermore, if we assume that the activation of the metaphor process or the literal process is maintained over some period of time, then we would expect that processing a sentence as a metaphor or as a literal can be affected by the metaphorical or literal nature of the prior sentence. If the prior context sentence were metaphoric, then we would expect facilitation in processing the target sentence if it, too, were metaphoric. Similarly, a literal prior context sentence would facilitate the processing of a literal target sentence. This hypothesis will be referred to as the "process priming" hypothesis.

Experiment 3 addressed these issues. Only short context (one sentence) was provided for each target sentence, but every context sentence was designed to make apparent the correct interpretation of the following target sentence. The target sentence contained a critical word that was to be interpreted either literally or metaphorically, depending on prior context. Assuming that metaphor processing is as efficient as literal processing when sufficiently supportive prior context is provided, we would predict equivalent reading times for literal and metaphoric target sentences. If the length of prior context is critical, however, then metaphoric targets will take longer to comprehend than literal targets (as in Experiment 1), because only short context is provided.

Most importantly, Experiment 3 provided a test of the process-priming hypothesis. Consider the possible pairings of target sentences (12) and (13) with the prior context sentences (10) and (11):

(10) the company used competitive tactics.

(11) the company used murderous tactics.

(12) the directors mercilessly outbid smaller companies.

(13) the directors mercilessly choked smaller companies.

Under the process-priming hypothesis, reading the metaphoric sentence (13) should be facilitated if it follows the 
metaphoric context sentence (11), relative to when it follows the literal context sentence (10). Reading the literal target (12) should be facilitated by prior context (10), relative to prior context (11). These facilitation effects may be observed even in the absence of a difference in the overall reading times of (12) and (13).

\section{Method}

\author{
Subjects \\ Twelve University of Massachusetts students were paid to participate. \\ All subjects had normal vision.
}

\section{Materials}

The stimuli consisted of 30 passages, each passage consisting of two sentences. The first sentence was the context sentence, and the second was the target sentence. Sentences ranged from 5 to 13 words in length, covering one or two lines on the CRT, so that passage length ranged from two to four lines.

There were six conditions in all. Four conditions were based on the four possible pairings of the literal and metaphoric versions of a context sentence with the literal and metaphoric versions of a target sentence. For example, context sentences (10) and (11) were paired with target sentences (12) and (13), to yield passages (10-12), (10-13), (11-12), and $(11-13)$, corresponding to the literal-literal, literal-metaphor, metaphor-literal, and metaphor-metaphor conditions. Each passage formed a thematically coherent unit. The two versions of a context sentence were identical except that a literal expression in the literal version was replaced with a metaphoric expression in the metaphoric version. The two versions of the target sentence were identical except for one critical word. The literal version of a target sentence contained a critical word that was to be interpreted literally, whereas the metaphoric version of a target sentence contained a critical word that was to be interpreted metaphorically. Critical words in a target sentence pair were equated for length whenever possible; the average critical word length is 6.8 letters for both the literal targets and the metaphoric targets. None of the critical words occupied initial or final positions in a sentence. Critical words were approximately matched on word frequency: The average frequency of literal critical words is 39 per million, and the average frequency of metaphoric critical words is 27 per million (Kučera \& Francis, 1967).

In addition, two "associated-word" context conditions were included. Sentence 14 is the associated-word context for target sentences (12) and (13):

(14) He was accused of murderous assault.

The associated-word context sentence contained a word identical to a word in the corresponding metaphoric context sentence; for example, (14) and (11) both contain the word murderous. However, the word in the associated-word context sentence was to be interpreted literally, whereas the same word in the metaphoric context sentence was to be interpreted metaphorically. For example, associated-word context sentence (14) suggests the literal interpretation of murderous, whereas metaphoric context sentence (11) suggests a metaphoric interpretation. Unlike the literal and metaphoric context, the associated-word context was not thematically related to the topic of the target sentence; passages (14-12) and (14-13), unlike passages (10-12), (10-13), (11-12), and (11-13), do not form conceptually coherent units. Stimuli are listed in Appendix.

The associated-word condition was included to test the possibility that any facilitation observed in the metaphor context-metaphor target passages (compared with the literal context-metaphor target passages) is due simply to a semantic priming effect resulting from the relationship between the literal sense of a salient word in the context sentence and the literal sense of the critical word in the target sentence. If any observed facilitation were due mainly to such priming of semantic associates, then target sentence reading times in the associated-word context-metaphor target condition should be similar to those in the metaphor context-metaphor target condition. For example, both associated-word context sentence (14) and metaphor context sentence (11) contain the word murderous, which is likely to be associated in semantic memory with the critical word of target sentence (13), choked. Similar target sentence reading times should be observed in passages (14-13) and (11-13) if semantic priming of literal meanings is responsible for the facilitation of (11-13) over (12-13).

The associated-word contexts also provide a check on whether or not subjects use prior context to establish a conceptual framework within which to interpret the target sentence. Because an associated-word context sentence is thematically unrelated to the target sentence it precedes, it should lead to the building of a framework inappropriate for target comprehension. This difficulty should reveal itself in an increase in target sentence reading time in the associated-word conditions.

\section{Design}

Two factors, (1) context (literal, metaphoric, and associated word) and (2) target (literal and metaphoric) were varied orthogonally. Six lists were constructed, each containing one of the six possible contexttarget versions of each passage (see Appendix). Within each list, the passages were presented in random order, so that the six conditions were varied within subjects and list constituted a between-subjects variable. Data were analyzed in 6 (list) $\times 3$ (context) $\times 2$ (target) ANOVAS. Dependent variables included target sentence reading time, context sentence reading time, and total viewing time of the critical word in the target sentence. Regressive eye movements were also informally examined.

\section{Procedure}

After two-dimensional calibration as in Experiment 2, each passage was read in the following manner: An initial fixation marker was displayed at the top left-hand side of the CRT. This position coincided with the first letter position of the first word on the top line of text. Upon having read the passage, the subject pressed a button that replaced the text with the fixation marker. Occasionally, readers were asked to paraphrase the passage they had just read. The subjects were not informed about the nature of the relationship between the two sentences in a passage.

An experimental session consisted of 36 passages. The initial 6 passages were training trials. Experimental data were collected from the remaining 30 passages. A complete session lasted about $1.5 \mathrm{~h}$.

\section{Results and Discussion}

\section{Target Sentences}

Average reading times of the target sentences are shown in Table 1 . There was virtually no evidence that metaphoric target sentences $(1,980 \mathrm{msec})$ required longer reading times than literal target sentences $(1,943 \mathrm{msec})$. Prior context, on the other hand, significantly affected target reading time $[F(2,12)=7.40, p<.01]$. Newman-Keuls tests showed that associated-word context yielded longer target sentence reading times $(2,167 \mathrm{msec})$ than literal and metaphoric context $(1,853$ and $1,864 \mathrm{msec})(\mathrm{q}=4.79$, $p<.05 ; q=4.62, p<.05$ ), which suggests that readers established a conceptual frame of reference during the reading of the context sentence within which the target information was interpreted. If this had not been the case, then the associated-word condition target sentence reading times would have been similar to the metaphor context condition and literal context condition target sentence reading times. (The associated-word passages did not form thematically coherent units.)

An additional context (literal, metaphoric) $\times$ target (literal, metaphoric) ANOVA was performed on the target sentence reading times to test, first, whether differ- 
Table 1

Sentence Reading Times (in Milliseconds) of the Target Sentence, Experiment 3 (The Corresponding Reading Times of the Context Sentences Are Presented in Parentheses)

\begin{tabular}{cccc}
\hline & \multicolumn{3}{c}{ Context Sentence } \\
\cline { 2 - 4 } Target Sentence & Associated & Literal & Metaphor \\
\hline Literal & $2194(1788)$ & $1742(2032)$ & $1892(2226)$ \\
Metaphor & $2140(1704)$ & $1963(2189)$ & $1836(2386)$ \\
\hline
\end{tabular}

ent processing strategies are activated during the comprehension of literal and metaphoric sentences and, second, whether the conceptual relationship between context and target was sufficient to yield equivalent processing times of metaphoric and literal target sentences despite the brevity of the context. The results showed that metaphoric target sentences were read faster when preceded by metaphoric context $(1,836 \mathrm{msec})$ than when preceded by literal context $(1,963 \mathrm{msec})$. The faster reading times in the metaphor-metaphor condition than in the literal-metaphor condition were not due to semantic associations between the literal meaning of a salient word in the metaphoric context sentence and the critical word in the metaphoric target sentence. This explanation is ruled out by the finding that the associated-word context yielded much longer target sentence reading times than did the metaphoric context. In addition, literal target sentences were read faster when preceded by literal context $(1,742 \mathrm{msec})$ than when preceded by metaphoric context $(1,892 \mathrm{msec})$. The context (literal, metaphoric) $\times$ target (literal, metaphoric) interaction was significant $[F(1,6)=6.67, p<.05]$.

A comparison of the reading times of literal and metaphoric target sentences showed somewhat shorter reading times for literal than for metaphoric sentences $(1,817$ and $1,900 \mathrm{msec}$, respectively), but this difference was not statistically reliable $[F(1,6)=1.18, p<.30]$. The similarity in target reading times for literal sentences and metaphoric sentences suggests that the literal and metaphoric contexts provided in this experiment, although short, were sufficient to allow correct interpretation of both literal and metaphoric target sentences.

Analysis of total viewing times of critical words in target sentences yielded a pattern similar to the target sentence reading time results. (See Table 2.) This analysis must be interpreted with caution, however, because the metaphoricity of a critical word was not necessarily obvious when the word itself was encountered. On some trials, the subjects had to read the target sentence in its entirety before the metaphoricity of the critical word became apparent. ${ }^{1}$ As in the analysis of target sentence reading times, there were somewhat shorter total viewing

Table 2

Total Viewing Time (in Milliseconds) of the Critical Word of the Target Sentence, Experiment 3

\begin{tabular}{cccc}
\hline & \multicolumn{3}{c}{ Context Sentence } \\
\cline { 2 - 4 } Target Sentence & Associated & Literal & Metaphor \\
\hline Literal & 357 & 279 & 309 \\
Metaphor & 342 & 329 & 307 \\
\hline
\end{tabular}

times on literal critical words than on metaphoric critical words (294 and $318 \mathrm{msec}$, respectively); again, the difference was not statistically reliable $[F(1,6)=2.12$, $\mathrm{p}<.20]$.

\section{Regressive Eye Movements}

An informal inspection of readers' regressive eye movements was revealing. This inspection considered only the first regressive eye movement each subject made within each target sentence. ${ }^{2}$ Literal targets that followed literal context accounted for $12 \%$ of the first regressions, and metaphoric targets that followed metaphoric context accounted for $11 \%$. Literal targets that followed metaphoric context accounted for $15 \%$ of first regressions; metaphoric targets that followed literal context accounted for $22 \%$. (The remaining $40 \%$ of first regressions occurred in the associated-word conditions.) Thus, the observed proportions of first regressions within a target sentence are consistent with the process-priming hypothesis. Interestingly, however, these results also show that regressions were initiated more frequently during the reading of metaphoric target sentences, which suggests, consistent with the stage model, that a reinterpretation occurred.

\section{Context Sentences}

The sentence reading times of literal and metaphoric context sentences were analyzed in a 2 (context: literal vs. metaphoric) $\times 2$ (target: literal vs. metaphoric) ANOVA. Interestingly, there were longer context sentence reading times in the metaphoric context condition than in the literal context condition (2,307 and $2,111 \mathrm{msec}$, respectively). This difference was statistically reliable $[F(1,6)=7.84, p<.05]$. No other effect reached statistical significance (all ps $<.1$ ). Thus, comprehending a metaphor required more time than comprehending a literal when no context had yet been provided, as in reading the very first sentence of a passage. However, once a conceptual frame of reference had been established, as in the reading of the second (target) sentence, metaphor comprehension did not take significantly more time than comprehension of literals.

\section{GENERAL DISCUSSION}

Conceptually supportive context is an important factor in the comprehension of metaphoric language. The results of Experiments 1 and 2 stand in empirical agreement with those of Ortony et al. (1978): Sentences that were to be interpreted metaphorically were comprehended more slowly than those that were to be interpreted literally only when contextual support was minimal. When context was extensive, metaphor comprehension was about as quick as literal comprehension.

The schema theory offered by Ortony et al. (1978) can explain these results. The finding that long context leads to an advantage of literal and metaphoric targets over unrelated targets can be accommodated by assuming that the schemata needed for interpreting the unrelated targets 
were absent from the antecedent context. The schema model can also handle the finding that, under short context, metaphoric targets and unrelated targets were read in about the same amount of time, whereas literal targets took less time to read. The schemata needed for correctly interpreting the metaphoric target were not activated by the minimal prior context available, just as the schemata needed for interpreting an unrelated target were absent.

Although the schema model as sketched above neatly handles the results of Experiments 1 and 2, it would need refinement to account for the results of Experiment 3. In Experiment 3, we found evidence that even minimal context can be sufficient to support the correct interpretation of metaphoric as well as literal sentences. If this had not been true, then the thematically unrelated prior context would not have led to longer target reading times than the thematically related prior contexts. It seems, then, that even in circumstances in which few schemata have been activated by the time the target sentence is read, metaphors can be comprehended about as easily as literals. How can the schema model explain this result? An important observation that may account for the context effects of Experiment 3 is that the metaphors used in Experiment 3 differed in kind from those used in Experiment 1. The metaphoric targets in Experiment 1 were sentences whose metaphoric interpretations were very much tied to what had gone before. For example, metaphoric interpretation of (3) with no prior context at all would be impossible. In contrast, the metaphoric targets used in Experiment 3 often had no literal sense at all, or a literal sense that seems semantically unlikely. In addition, Experiment 3 passages maintained a great deal of broadly defined referential overlap (see Appendix). To use Haviland and Clark's (1974) terminology, the relationship between the given information and the new information is readily transparent in Experiment 3 , so that context could be used effectively during the reading of the target sentence.

We have seen that schema theory captures two factors that influence ease of metaphor comprehension, length of context and degree of transparency of the relationship between context and target. Another finding of Experiment 3, however, remains to be explained: Metaphoric target sentences were comprehended faster when they followed metaphor context sentences than when they followed literal context sentences, and, correspondingly, literal targets were read faster when they followed literal contexts than when they followed metaphoric contexts. The process priming hypothesis states that this context $x$ target interaction reflects the existence of a metaphor processing strategy different from the literal processing strategy. The processing strategy that is activated tends to remain activated for some time, so that reading a metaphor tends to "prime" interpreting the subsequent sentence metaphorically, whereas reading a literal primes literal interpretation of the subsequent sentence. Can schema theory account for the context $\times$ target interaction without the additional assumption of process priming? The schema theory could claim that the metaphor- metaphor and literal-literal passages tended to have more schema overlap than did the literal-metaphor and metaphor-literal passages, and an inspection of the passages lends some support to this claim.

We did an additional analysis of the data of Experiment 3 in order to test whether a pure process-priming hypothesis can account for the interaction. We reasoned that if the process-priming hypothesis were correct, then there should be interpassage facilitation effects as well as intrapassage effects; that is, the processing of a metaphoric context sentence should be facilitated by a metaphoric target sentence in the previously presented passage. The schema explanation, in contrast, would predict no effects between adjacent passages, because the schemata involved in comprehending the context sentence in passage " $x$ " are entirely different from the schemata involved in comprehending the target sentence in passage " $x-1$." A comparison of the effects of metaphoric and literal target sentences on the reading times for metaphoric context sentences of the following passage yielded reading times of 2,098 and 2,284 msec, respectively. Since context sentences were not matched for length, an additional comparison was performed on the per-word reading times for metaphoric context sentences, revealing that metaphoric contexts immediately preceded by metaphoric targets were read at $245 \mathrm{msec}$ per word, whereas those preceded by literal targets were read at $264 \mathrm{msec}$ per word. However, statistical analyses showed these differences to be unreliable $(\mathrm{t}<1)$.

Thus, we are lead to conclude that the schema model can account for the results of the present experiments. However, it seems to us that a rejection of the processpriming hypothesis would be premature. The possibility remains that process priming is effective within a relatively short time interval. When time intervals are relatively long, as they are between presentations of successive text passages, the effects of process priming may become diluted.

We have seen that adequate contextual support leads to quick target interpretation even when the target is expressed metaphorically. Gildea and Glucksberg (1983) suggested that the processing of a metaphor is analogous to the processing of an ambiguous word; in both cases, the correct context-dependent interpretation emerges automatically. However, unlike the disambiguation of an ambiguous word, during which the contextually incorrect interpretation rarely reaches awareness, reading a metaphor does sometimes invoke awareness of the literal as well as the metaphoric meaning. The concurrent awareness of the two meanings of a metaphoric expression is an interesting phenomenon, and it invites further investigation.

\section{REFERENCES}

Carpenter, P. A., Daneman, M. (1981). Lexical retrieval and error recovery in reading: A model based on eye fixations. Journal of Verbal Learning and Verbal Behavior, 20, 137-160.

Cassirer, E. (1923). Substance and function. Chicago: Open Court. 
GildeA, P., \& Glucksberg, S. (1983). On understanding metaphors: The role of context. Journal of Verbal Learning and Verbal Behavior, 22, 577-590.

Haviland, S. E., \& Clark, H. H. (1974). What's new? Acquiring new information as a process in comprehension. Journal of Verbal Learning and Verbal Behavior, 13, 512-521.

INHOFF, A. W. (1983). Attentional strategies during the reading of short stories. In K. Rayner (Ed.), Eye movements in reading: Perceptual and language processes. New York: Academic Press.

KoEN, F. (1965). An inter-verbal explication of the nature of metaphor. Journal of Verbal Learning and Verbal Behavior, 4, 129-133.

KuČERA, H., \& FrANCIs, W. N. (1967). Computational analysis of present-day American English. Providence, RI: Brown University Press.

Ortony, A., Schallert, D. L., Reynolds, R. E., \& Antos, S. J. (1978). Interpreting metaphors and idioms: Some effects of contex upon comprehension. Journal of Verbal Learning and Verbal Behavior, 17, 465-477.

Rayner, K., Inhoff, A. W., Morrison, R. E., Slowiaczek, M. L., \& BerterA, J. H. (1981). Masking of foveal and parafoveal vision during eye fixations in reading. Journal of Experimental Psychology: Human Perception and Performance, 7, 167-179.
RicharDs, I. A. (1936). The philosophy of rhetoric. London: Oxford University Press.

Searle, J. R. (1969). Speech acts. London: Cambridge University Press.

Searle, J. R. (1979). Metaphor. In A. Ortony (Ed.), Metaphor and thought. London: Cambridge University Press.

VerbrugGe, R. R., \& MCCarRell, N. S. (1977). Metaphoric comprehension studies in reminding and resembling. Cognitive Psychology, $9,494-533$.

\section{NOTES}

1. Carpenter and Daneman (1981) showed that an incorrect assessment of individual words is often accompanied by a regression to the incorrectly accessed word. Total viewing time, which includes regressive fixation time, includes processing time that may be due to reinterpretation of initially misinterpreted words. Such reinterpretations may occur when the metaphoricity of a particular critical target word was not apparent during its initial reading.

2. Only intratarget sentence regressions longer than three character spaces were considered. The regression data reported are from 11 subjects only. The regressions of one subject were discarded because of this subject's tendency to reread context and target sentences.

Appendix

Stimuli Used in Experiment 3

(1) A: The insect approached its prey.

C: The jumbo jet was a big machine waiting for the travellers.
insect

T: The airplane $\begin{aligned} & \text { contained } \\ & \text { swallowed }\end{aligned}$

(2) A: He used to dance.

C: Heat waves made the highway flicker.

$\mathrm{T}$ : The road became a shapeless line.

(3) A: We heard an orchestra before dinner.

C: The gourmet's kitchen held a collection of pots and pans.

T: The teapot was boiling on the stove.

(4) A: The old house had stained-glass windows.

C: The light through the trees reminded me of the moment before sunset.

T: We walked through the $\begin{aligned} & \text { forest } \\ & \text { church }\end{aligned}$ of pines.

(5) A: He decided to replace the fabric on the cushion of his favorite chair.

C: The new vice-president acted as a listener for the union's complaints.

$\mathrm{T}$ : He was hired as a helper for the president.

(6) A: His face was all smiles yesterday.

C: The days had lost their $\begin{aligned} & \text { appeal } \\ & \text { smiles }\end{aligned}$

$\mathrm{T}$ : The cynic loathed his frowned upon his life.

(7) A: We slept under a white blanket.

C: After the blizzard, the valley $\begin{aligned} & \text { was put } \\ & \text { slept }\end{aligned}$

T: This snow formed a fresh $\frac{\text { layer }}{\text { sheet }}$ on the land. 
(8) A: The hay lay in spots on the field.
C: The sunshine fell on part of
$\mathrm{T}$ : The sky was $\begin{aligned} & \text { clouded } \\ & \text { freckled }\end{aligned}$

(9) A: The child was sad and depressed.

C: The sky was dark and cloudy.

T: The sad and depressed.

$\mathrm{T}$ : We took shelter as the tears began to fall from the sky.

(10) A: We pushed the big rocks out of our way.

C: The guard stood motionless at the door.

$\mathrm{T}$ : No one could get past this $\begin{aligned} & \text { soldier } \\ & \text { boulder }\end{aligned}$ to see the dictator.

(11) A: The crowned heirs ruled over the country.

C: The snow covered mountains $\begin{aligned} & \text { blocked the valley. } \\ & \text { rnow crowned } \\ & \text { hills }\end{aligned}$
T: These tall kings reached the clouds.

(12) A: The lion was in the lion tamer's cage.

C: The army camp was a training ground.

lion tamer's cage.

T: Occasionally, the sergeant $\frac{\text { directed }}{\text { whipped }}$ the recruits with his commands

(13) A: Her sewing needle was broken.

C: Rain came down in incessant $\begin{aligned} & \text { streams } \\ & \text { needles }\end{aligned}$

$\mathrm{T}$ : Drop after drop $\begin{aligned} & \text { touched } \\ & \text { pricked }\end{aligned}$ the surface of the earth.

(14) A: Some tea was poured into the glass.

C: Work was over and people $\frac{\text { amassed in }}{\text { poured into }}$ the streets.

T: The city was a bustling ocean at rush hour.

(15) A: He was accused of murderous assault.

C: The company used competitive tactics.

T: The directors mercilessly $\begin{aligned} & \text { outbid smaller companies. } \\ & \text { choked }\end{aligned}$

(16) A: The governor outlawed dueling.

C: The fish faced each other as if they were attached.

T: John watched the fish float fonce a while.

(17) A: His mother pounded the roach with the sole of her shoe.

C: The professor lectured to the students with eloquence

T: These persuasive notions were not quickly forgotten.

(18) A: The little plants were beginning to erupt through the soil.

C: His sister knew he was so angry, he was about to $\begin{aligned} & \text { scream violently. } \\ & \text { erupt }\end{aligned}$

T: She tried to placate the volcano as best as she could.

(19) A: The old woman sighed loudly.

C: The jazz clarinet $\begin{aligned} & \text { played } \\ & \text { sighed }\end{aligned}$ sad sounds.

T: The band $\begin{aligned} & \text { delivered } \\ & \text { whispered }\end{aligned}$ a slow blues song. 
(20) A: The sea flooded the bay.

C. The crowded

C: The station was flooded with people

T: Each time a train arrived a mass of passengers left the station.

(21) A: She whispered in his ear

C: The man wore a thin ${ }_{\text {whisper of a }}$ white beard.

$\mathrm{T}$ : His hair was an intensive red color.

(22) A: The killer suffocated his victim

C: The sand storm blew across the prairie.

$\mathrm{T}$ : The day $\underset{\text { died }}{\text { ended }}$ in a pall of dust.

(23) A: The attorney discussed the case of a sentenced killer.

C: The children complained that they were $\begin{aligned} & \text { compelled to study. } \\ & \text { sentenced }\end{aligned}$

T: They came to their $\begin{aligned} & \text { schools } \\ & \text { prisons }\end{aligned}$

(24) A: The bewitched frog turned into a prince.

C: The candidate bewitched his listeners.

$\mathrm{T}$ : This sorcerer won the election with ease.

(25) A: We were kept awake all night by some dogs baying at the moon.

C: The man's voice sounded like he was serenading his lover

$\mathrm{T}$ : The performer was romantically singing an old tune.

(26) A: A snowstorm damaged the crops.

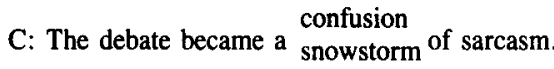

$\mathrm{T}$ : He could see no purpose to wait for the ridiculous $\begin{gathered}\text { argument } \\ \text { blizzard }\end{gathered}$

(27) A: The coals were red hot when we started cooking the chicken.

C: The woman spoke of the traitor with $\begin{aligned} & \text { outraged passion at the meeting. } \\ & \text { red hot fire }\end{aligned}$

T: Her angry words $\begin{aligned} & \text { accused } \\ & \text { branded }\end{aligned}$ him as a murderer.

(28) A: The antidote for the venom was very old.

C: The criminal's words were filled with lies venom and spite.

$\mathrm{T}$ : The judge sent the scowling $\begin{aligned} & \text { thief } \\ & \text { snake }\end{aligned}$ to jail.

(29) A: The villagers lived in constant fear of the army of invaders.

C: The farmers feared the ants would be $\begin{aligned} & \text { a source } \\ & \text { an army }\end{aligned}$

T: Silently the insects $\begin{aligned} & \text { crawled } \\ & \text { marched }\end{aligned}$ toward the crops.

(30) A: She was concerned about her face.

C: Advertising has an ugly face, too.

$\mathrm{T}$ : Billboards are $\begin{aligned} & \text { signs } \\ & \text { warts }\end{aligned}$ 